See discussions, stats, and author profiles for this publication at: https://www.researchgate.net/publication/265731924

\title{
Engineering nonlinear response of nanomaterials using Fano resonances
}

Article in Journal of optics · September 2014

DOI: 10.1088/2040-8978/16/10/105009

\section{CITATIONS}

13

4 authors, including:

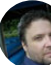

Gürsoy b Akgüç

Izmir Ekonomi Üniversitesi

41 PUBLICATIONS 244 CITATIONS

SEE PROFILE

7. Mehmet Emre Tasgin

Hacettepe University

68 PUBLICATIONS 203 CITATIONS

SEE PROFILE

Some of the authors of this publication are also working on these related projects:

Crystallization of a-Si Thin Films via Nanosecond Pulsed Laser View project

Project Novel methods for enhanced Raman spectroscopy View project
Alpan Bek

Middle East Technical University

106 PUBlications 1,214 CITATIONS

SEE PROFILE 


\title{
Engineering the nonlinear response of a metal nanoparticle dimer using the Electromagnetically Induced Trasparency (EIT)
}

\author{
Deniz Türkpençe, ${ }^{1}$ Alpan Bek, ${ }^{2}$ and Mehmet Emre Taşgın ${ }^{1,3,4}$ \\ ${ }^{1}$ Department of Electrical \& Electronics Engineering, \\ Kirklareli University, 39100 Kayalı, Kirklareli, Turkey \\ ${ }^{2}$ Department of Physics, Middle East Technical University, 06800, Ankara, Turkey \\ ${ }^{3}$ Department of Physics, Bilkent University, 06800, Ankara, Turkey \\ ${ }^{4}$ to whom correspondence should be addressed.
}

(Dated: November 4, 2013)

\begin{abstract}
We show that, nonlinear optical processes in a plasmonic metal nanoparticle (MNP) dimer can be controlled by the presence of a molecule or a quantum dot. (i) Frequency conversion can be suppressed if the dimer is coupled to a quantum object which is resonant to the generated frequency. This occurs simply because, EIT does not allow an excitation at the converted frequency frequency. (ii) On the contrary, a similar effect can be used to enhance the frequency conversion. Nonlinear processes can be brought to resonance without tuning the dimer modes. Path interference effect cancels the nonresonant frequency terms. Here, we consider the second harmonic generation (SHG) as an example to demonstrate the control mechanism. However, the method can be easily generalized to other nonlinear processes.
\end{abstract}

PACS numbers:

\section{INTRODUCTION}

Resonant interaction of metal nanoparticles (MNPs) with optical light provides a tool for the strong localization of electromagnetic field [1]. Intensity enhancements as high as $10^{5}$ can be achieved $[1,2]$ within the surface plasmon-polariton (SPP) modes, in terms of coupled oscillators of surface electrons and the localized optical field [3]. Such an orders of magnitude increase in the intensity leads to the emergence of optical nonlinearities [4] , e.g. enhanced Raman scattering [5], four wave mixing [6] and second harmonic generation [7-10].

Emergence of nonlinear processes can be both desirable or unwanted depending on the operating properties of the manufactured device. In example, for higher resolution the nonlinear processes called the surface-enhanced Raman scattering is required [11]. The nonlinear response of the media can also be utilized for optical switching [12]. The second harmonic generation (SHG) process can enhance the absorption efficiency in Photovoltaic devices [13], may increase the coherence time (length) of the field [14-16] as well as being able to generate entangled photon pairs [17].

On the other hand, nonlinear conversion may be undesirable in other devises. Raman scattering process in fiber-optic cables limits the number of channels that could be used for a given bandwidth [18-21]. Similarly nonlinear effects can decrease the quality factor of microwave cavities $[22,23]$. In addition, one may require the operation of her/his device in the linear regime even for higher input powers. Because nonlinearities may cause unexpected chaotic behavior for the long term operation [24].

Besides the emergence of nonlinearities, analog of electromagnetically induced transparency [25] has also been observed in plasmonic excitations of MNPs [2, 26-34].
The attachment of a molecule/quantum dot to a MNP strongly modifies the optical response of the hybrid material $[2,16,35-38]$. The presence of a quantum object with small decay rate introduces two possible paths for the absorption/polarization of the incident light. At frequencies resonant to level spacing of the quantum object $\left(\omega \cong \omega_{e g}\right.$ ) the two paths for the absorption of the photon interfere destructively [25, 39]. There emerges a transparency window centered about $\omega_{e g}$, where polarization of MNP-quantum object hybrid system is avoided [16].

In this paper, we show that it is possible to manage the nonlinear behavior of a material using the path interference effects. We place a quantum dot/molecule at the hot-spot of a MNP dimer (see Fig. 1, top) which has a low decay rate $\left(\gamma_{e g}\right)$ compared to the $\mathrm{MNP}[26]\left(\gamma_{1,2}\right)$. The dimer has surface plasmon-polariton (PP) resonances $\omega_{1}$ and $\omega_{2}$ (see Fig.1, bottom) for the polarization. The drive frequency $\omega$ and the second harmonic $(\mathrm{SH})$ frequency $2 \omega$ fall into the excitation range of the $\omega_{1}$ and $\omega_{2}$ polarization modes, respectively. Without the presence of the quantum emitter, resonance of the SHG process occurs when $\omega_{1}=\omega$ and $\omega_{2}=2 \omega$ (see the discussion in Sec. IIB). We show that, (i) even in the resonance condition for $\mathrm{SH}$ conversion (that is $\omega_{1}=\omega, \omega_{2}=2 \omega$ ), the presence of coupling to the quantum emitter can suppress the nonlinear process down to 9 orders of magnitude (see Fig. 2). This effect is observed, because cancellation of the two excitation paths does not allow polarization in the $\omega_{2}$ $\mathrm{PP}$ mode of the MNP dimer. Suppression is maximum when quantum level spacing is resonant to conversion frequency, $\omega_{e g}=2 \omega$. (ii) On the other hand, a similar path cancellation effect can be adopted to kill the nonresonant term $\left[\left(\omega_{2}-2 \omega\right)\right]$ that emerges when $\omega_{2}$ is not resonant to the SHG frequency $2 \omega$. Without adjusting the resonances [40] of the dimer $\left(\omega_{1}, \omega_{2}\right)$, the SHG process can be carried closer to resonance (Fig. 4). These two effects 
together, enables the control over induction of the nonlinearities without the need for managing the properties of the material.

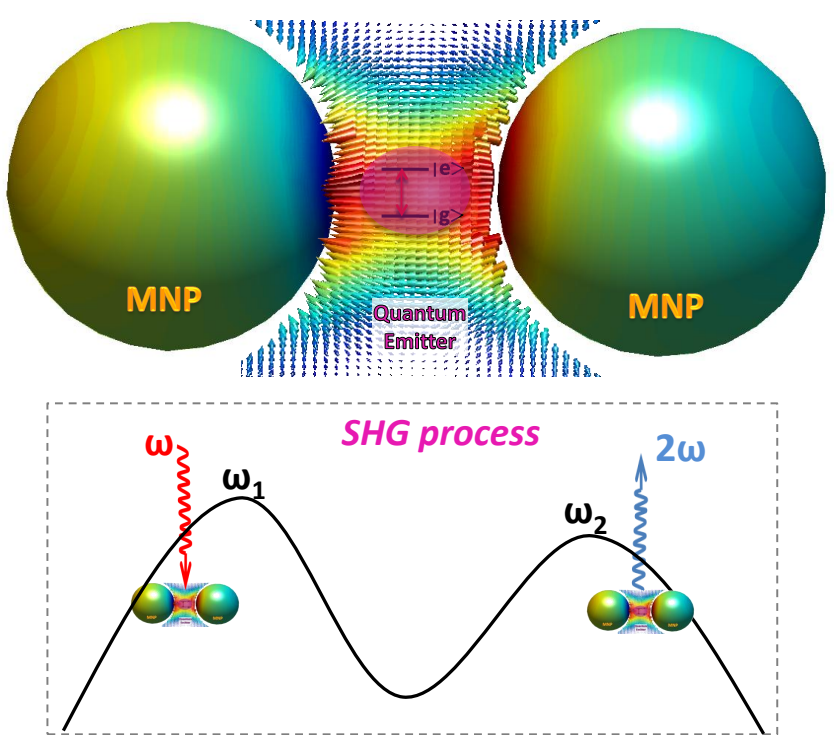

FIG. 1: Top: A quantum emitter (purple) with a small decay rate is placed at the center of a MNP dimer [26]. The polarization of the plasmon-polariton (PP) modes strongly localizes the incident field to the center (see the field vectors). Field enhancement gives rise nonlinear processes, e.g. second harmonic generation (SHG). Bottom: The incident planewave field $\left(\epsilon_{p} e^{-i \omega t}\right)$ drives the $\hat{a}_{1} \mathrm{PP}$ polarization mode (resonance $\left.\omega_{1}\right)$ of the dimer. The intense localized polarization field of $\hat{a}_{1}$, oscillating with $\omega$, gives rise to SHG [43]. This process induces polarization oscillations $\left(e^{-i 2 \omega t}\right)$ in the second PP mode $\hat{a}_{2}$ whose resonance is $\omega_{2}$. The quantum object (level spacing $\left.\omega_{e g} \simeq 2 \omega\right)$ interacts with the field of the $\hat{a}_{2}$ polarization mode. Quantum emitter is chosen to have no SH response [57] to $\omega$. The observation of the $\mathrm{SH}$ light occurs due to the radiative decay of the $\hat{a}_{2} \mathrm{PP}$ mode $[43,54-56]$.

Here, we present the method for the second harmonic generation process in a MNP dimer. However, method can be generalized to other nonlinear frequency generation processes as long as the two modes of the material can be resolved (see Sec. IID). Emergence of the suppression is a quite general phenomenon independent of the details of the system (Sec. IID).

The paper is organized as follows. In Sec. II A, we describe the SHG process in the coupled system of a MNP dimer and a quantum emitter. We introduce the Hamiltonian for the hybrid system. Nonlinear frequency conversion process is included in the second quantized Hamiltonian. We derive the equations of motion for the system using the density matrix formalism for the quantum object. We include the damping and quantum decay rates and the source driving the MNP dimer. In Sec. II B, we demonstrate that conversion process is suppressed for $\omega_{e g} \simeq 2 \omega$. In Sec. II C, we present a contrary effect. The cancellation of the nonresonant terms leads to enhanced production of the SHG for the choice of $\omega_{e g} \simeq 2.013 \omega$.
Sec. III includes our conclusions.

\section{MODIFICATION OF THE NONLINEAR RESPONSE}

In this section, we describe the response of a coupled MNP dimer-quantum object system to a driving electromagnetic field. We shortly mention about the nature of couplings in the hybrid system and the mechanism for SHG on the MNP-dimer resonator.

We give the effective Hamiltonian for the system and drive the equations of motion for the fields of the plasmon-polariton modes together with the excitation of the quantum emitter. We find the equations governing the steady state values of the excitations to obtain the linear behavior of the hybrid system. Using these equations, we demonstrate the principle behind gaining control over the process of nonlinear frequency generation. We show that by choosing the appropriate level spacing $\left(\omega_{e g}\right)$ for the quantum emitter, one can either suppress and enhance the non-linear frequency generation.

\section{A. Hamiltonian and equations of motion}

We consider a system where a quantum object (e.g. quantum dot [41], molecule [42] or a nitrogen-vacancy center $[37,38])$ is placed into the middle of the MNP dimer. The two MNPs can still be coupled for dimerization due to the small dimensions of the quantum object [26]. The two resonances of the MNP dimer $\omega_{1}$ and $\omega_{2}$ are relevant to the incident $(\omega)$ and $\mathrm{SH}(2 \omega)$ frequencies, respectively (see Fig. 1, bottom). The resonance frequency of the $\hat{a}_{2} \mathrm{PP}$ mode $\left(\omega_{2}\right)$ is about the SH frequency $2 \omega$, but not necessarily resonant to it.

The incident light, in the planewave mode with frequency $\omega$, couples strongly to the $\hat{a}_{1}$ plasmon-polariton mode of the dimer. The direct coupling of light to the quantum object is of negligible strength compared to the plasmon. Quantum emitter couples to the localized plasmon-polariton field of the dimer. The hot-spots for the both plasmon-polariton modes emerge in the middle of the two MNPs, where the quantum oscillator is tightly placed.

The dynamics of the total system is as follows. The incident planewave mode field $\left(\epsilon_{p} e^{-i \omega t}\right)$ drives the first dimer mode $\hat{a}_{1}$ (resonance $\omega_{1}$ ) at the oscillation frequency $\omega$. The polarization of the plasmon-polariton (PP) excitation yields a localized strong electromagnetic field mode $\left(\hat{a}_{1}\right)$ between the two MNPs. Such an enhancement in the field gives rise to the emergence of nonlinear effect (e.g. SHG) in the electron gas [26, 43-45]. Explicitly; the field (oscillating at $\omega$ ) trapped in the $\hat{a}_{1} \mathrm{PP}$ polarization gives rise to second harmonic polarization oscillations $(2 \omega)[26,43-53]$ in the $\hat{a}_{2} \mathrm{PP}$ polarization mode of the dimer. The quantum object, whose level spacing is compatible with the $\mathrm{SH}$ oscillation frequency 
$\omega_{e g} \simeq 2 \omega$, interacts with the polarization field of the $\hat{a}_{2} \mathrm{PP}$ mode. The resonance of $\hat{a}_{2}$ mode is $\omega_{2}$. The field localization at the hot-spot provides strong interaction with the quantum emitter. The SH light is observed through the radiative decay of the $\hat{a}_{2} \mathrm{PP}$ mode $[43,54-$ 56]. We assume that the molecule does not have a SHG response to the drive frequency $\omega$ [57].

Here, we consider an oversimplified model for the hybrid system. We mainly aim to demonstrate the principles behind the control mechanism. In more realistic calculations [29, 30], one has to consider complicating effects, such as the influence of the dielectric environment and exact spatial distribution of the fields. However, the oscillators model $[16,29]$ predicts the basic behavior of the MNPs combined with the quantum emitters $[35,37,38]$.

The total Hamiltonian $(\hat{H})$ for the described system can be written as the sum of the energy of the quantum object $\left(\hat{H}_{0}\right)$, energy of the plasmon-polariton oscillations $\left(\hat{a_{1}}, \hat{a_{2}}\right)$ of the MNP dimer $\left(H_{d}\right)$, the interaction of the quantum object with the plasmon-polariton modes $[16$, 26] $\left(H_{\text {int }}\right)$

$$
\begin{array}{r}
\hat{H}_{0}=\hbar \omega_{\mathrm{e}}|\mathrm{e}\rangle\left\langle\mathrm{e}\left|+\hbar \omega_{\mathrm{g}}\right| \mathrm{g}\right\rangle\langle\mathrm{g}|, \\
\hat{H}_{d}=\hbar \omega_{1} \hat{a}_{1}^{\dagger} \hat{a_{1}}+\hbar \omega_{2} \hat{a}_{2}^{\dagger} \hat{a_{2}}, \\
\hat{H}_{\text {int }}=\hbar\left(f_{c} \hat{a}_{2}^{\dagger}|\mathrm{g}\rangle\left\langle\mathrm{e}\left|+f_{c}^{*} \hat{a}_{2}\right| \mathrm{e}\right\rangle\langle\mathrm{g}|\right),
\end{array}
$$

as well as the energy transferred by the pump source $(\omega)$, $\hat{H}_{p}$ and the second harmonic generation process among the plasmon-polariton fields $\left(\hat{H}_{s h}\right)$

$$
\begin{gathered}
\hat{H}_{p}=i \hbar\left(\hat{a}_{1}^{\dagger} \epsilon_{p} e^{-i \omega t}-\hat{a}_{1} \epsilon_{p}^{*} e^{i \omega t}\right), \\
\hat{H}_{s h}=\hbar \chi^{(2)}\left(\hat{a}_{2}^{\dagger} \hat{a}_{1} \hat{a}_{1}+\hat{a}_{1}^{\dagger} \hat{a}_{1}^{\dagger} \hat{a}_{2}\right),
\end{gathered}
$$

respectively [25, 58]. In Eq. (1), $\hbar \omega_{e}\left(\hbar \omega_{g}\right)$ is the excited (ground) state energy of the quantum emitter. States $(|\mathrm{e}\rangle),|\mathrm{g}\rangle$ correspond to the (excited) ground levels of the quantum emitter. $\hat{a}_{1}, \hat{a}_{2}$ are the plasmon-polariton excitations induced on the MNP dimer and $\hbar \omega_{1}, \hbar \omega_{2}$ are the corresponding energies for the oscillation modes. $f_{c}$ is the coupling matrix element between the field induced by the $\hat{a}_{2}$ polarization mode of the MNP dimer and the quantum emitter. Eq. (4) describes the interaction of the light source (oscillations as $e^{-i \omega}$ ) driving the plasmonpolariton mode with smaller resonance frequency $\omega_{1}$. In Eq. (5), the fields of two excitations in the low-energy plasmon-polariton mode $\left(\hat{a}_{1}\right)$ combine to generate the field of a high energy plasmon-polariton polarization. Stronger the second generated plasmon-polariton excitation is, the detection of SHG photons $(2 \omega)$ increases. Because, $\hat{a}_{2}$ mode radiatively decay to $2 \omega$ photon mode due to the energy conservation in the input-output process $[43,54]$. The parameter $\chi^{(2)}$, in units of frequency, is proportional to the second harmonic susceptibility of the MNP dimer.

We note that, one could also treat the SHG process as originating directly from the incident field, e.g.
$\hat{H}_{s h} \sim\left(\hat{a}_{2}^{\dagger} \epsilon_{p}^{2} e^{-i 2 \omega t}+\right.$ c.c. $)$. Even though the following results would remain unaffected physically such a model would be inappropriate. Because, enhanced nonlinear processes emerge due to the electromagnetic field of the localized intense surface plasmon-polariton (polarization) mode $[43,54]$. However, the mode of the incident field $(\omega)$ is planewave.

We use the commutation relations (e.g. $i \hbar \dot{\hat{a}}=[\hat{a}, \hat{H}]$ ) in driving the equations of motions. We keep operators $\hat{a}_{1}, \hat{a}_{2}$ quantum up to a step in order to avoid any fault in the equations of motion. After obtaining the dynamics in the quantum approach, we carry $\hat{a}_{1}, \hat{a}_{2}$ to classical expectation values $\hat{a}_{1} \rightarrow \alpha_{1}, \hat{a}_{2} \rightarrow \alpha_{2}$. We introduce the decay rates for plasmon-polariton fields $\alpha_{1}, \alpha_{2}$. Quantum object is treated within the density matrix approach. The equations take the form

$$
\begin{array}{r}
\dot{\alpha}_{1}=\left(-i \omega_{1}-\gamma_{1}\right) \alpha_{1}-i 2 \chi^{(2)} \alpha_{1}^{*} \alpha_{2}+\epsilon_{p} e^{-i \omega t}, \\
\dot{\alpha}_{2}=\left(-i \omega_{2}-\gamma_{2}\right) \alpha_{2}-i \chi^{(2)} \alpha_{1}^{2}-i f_{c} \rho_{g e}, \\
\dot{\rho_{g e}}=\left(-i \omega_{e g}-\gamma_{e g}\right) \rho_{g e}+i f_{c} \alpha_{2}\left(\rho_{e e}-\rho_{g g}\right), \\
\dot{\rho_{e e}}=-\gamma_{e e} \rho_{e e}+i f_{c}\left(\alpha_{2}^{*} \rho_{g e}-\alpha_{2} \rho_{g e}^{*}\right),
\end{array}
$$

where $\gamma_{1}, \gamma_{2}$ are the damping rates of the MNP dimer modes $\alpha_{1}, \alpha_{2}$. $\gamma_{e e}$ and $\gamma_{e g}=\gamma_{e e} / 2$ are the diagonal and offdiagonal decay rates of the quantum object, respectively. To make a comparison, $\gamma_{1}, \gamma_{2} \sim 10^{14} \mathrm{~Hz}$ for MNPs [2] while $\gamma_{e e} \sim 10^{12}$ for molecules [35] and $\gamma_{e e} \sim 10^{9} \mathrm{~Hz}$ for quantum dots [28]. The constraint on the conservation of probability $\rho_{e e}+\rho_{g g}=1$ accompanies Eqs. (6a$6 d)$.

In our simulations (Figs. 2-4), we time-evolve Eqs. (6a$6 \mathrm{~d})$ numerically to obtain the long time behaviors of $\rho_{e g}$, $\rho_{e e}, \alpha_{1}$, and $\alpha_{2}$. We determine the values to where they converge when the drive is on for long enough times. We perform this evolution for different $\omega_{2}$ frequency values with the initial conditions $\rho_{e e}(t=0)=0, \rho_{e g}(0)=0$, $\alpha_{1}(0)=0, \alpha_{2}(0)=0$.

On the other hand, one may gain understanding about the linear behavior of Eqs. (6a-6d) by seeking solutions of the form

$$
\begin{gathered}
\alpha_{1}(t)=\tilde{\alpha}_{1} e^{-i \omega t} \quad, \quad \alpha_{2}(t)=\tilde{\alpha}_{2} e^{-i 2 \omega t}, \\
\rho_{e g}(t)=\tilde{\rho}_{e g} e^{-i 2 \omega t} \quad, \quad \rho_{e e}(t)=\tilde{\rho}_{e e}
\end{gathered}
$$

for the steady states of the oscillations. In our numerical simulations governing the time-evolution of Eqs. (6a-6d), we check that the solutions indeed converge to the form of Eq. (7) for long-time behavior. Inserting Eq. (7) into Eqs. (6a-6d), one obtains the equations for the steady state

$$
\begin{array}{r}
{\left[i\left(\omega_{1}-\omega\right)+\gamma_{1}\right] \alpha_{1}+i 2 \chi^{(2)} \alpha_{1}^{*} \alpha_{2}=\epsilon_{p},} \\
{\left[i\left(\omega_{2}-2 \omega\right)+\gamma_{2}\right] \alpha_{2}+i \chi^{(2)} \alpha_{1}^{2}=-i f_{c} \rho_{g e},} \\
{\left[i\left(\omega_{e g}-2 \omega\right)+\gamma_{e g}\right] \rho_{g e}=i f_{c} \alpha_{2}\left(\rho_{e e}-\rho_{g g}\right),} \\
\gamma_{e e} \rho_{e e}=i f_{c}\left(\alpha_{2}^{*} \rho_{g e}-\alpha_{2} \rho_{g e}^{*}\right),
\end{array}
$$

where $\tilde{\alpha}_{1}, \tilde{\alpha}_{2}, \tilde{\rho}_{g e}, \tilde{\rho}_{e e}$ are constants independent of the time. 


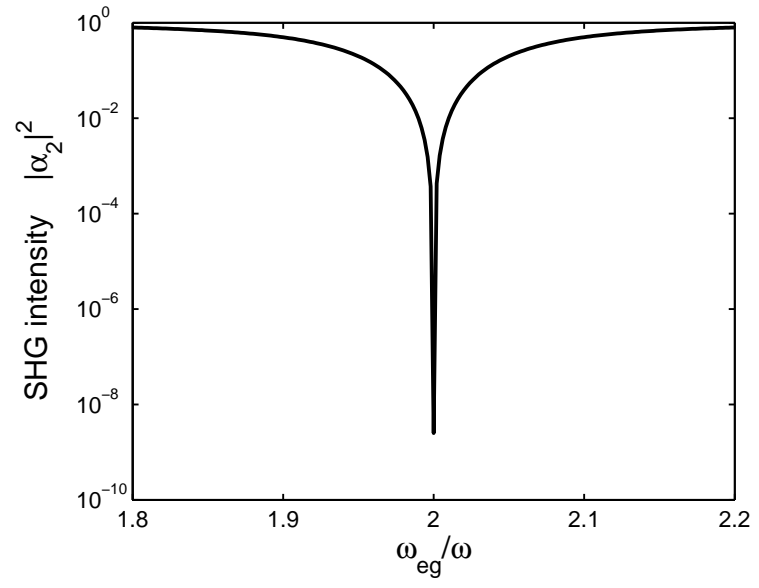

FIG. 2: Suppression of the SH polarization conversion to the $\hat{a}_{2}$ plasmon-polariton (PP) mode from the $\hat{a}_{1}$ mode. Even at the presence of resonant conversion condition, $\omega_{1}=\omega$ and $\omega_{2}=2 \omega$, the presence of quantum emitter $\left(\omega_{e g}=2 \omega\right)$ prevents the take place of the SHG process. EIT doesn't allow the polarization in the $\hat{a}_{2} \mathrm{PP}$ mode. The resonant conversion is represented by unity in the figure. When $\omega_{e g}=2 \omega$, the nonlinear intensity can be suppressed 9-orders of magnitude with respect to the resonant value. Decay rates are $\gamma_{1}=\gamma_{2}=0.1 \omega$ and $\gamma_{e g}=10^{-5} \omega$.

Using Eqs. (8b) and (8c), one can obtain

$$
\tilde{\alpha}_{2}=\frac{i \chi^{(2)}}{\frac{\left|f_{c}\right|^{2} y}{i\left(\omega_{e g}-2 \omega\right)+\gamma_{e g}}-\left[i\left(\omega_{2}-2 \omega\right)+\gamma_{2}\right]} \tilde{\alpha}_{1}^{2}
$$

for the steady value of $\hat{a}_{2}$ plasmon-polariton field mode. Here, $y=\rho_{e e}-\rho_{g g}$ is the steady state value which usually takes on values close to $y \cong-1$.

\section{B. Suppression of the nonlinear conversion process}

Taking a closer look at the denominator of Eq. (9), one can immediately realize that $\left|f_{c}\right|^{2} y / \gamma_{e g}$ attains huge values on resonance $\omega_{e g}=2 \omega$. Because, linewidth of the quantum emitter $\left(\gamma_{e g}\right)$ is very small compared to all other frequencies. If $f_{c} \neq 0$, the largeness of the $\left|f_{c}\right|^{2} y / \gamma_{e g}$ term dominates the denominator. This results in the suppression of the generation of the $\tilde{\alpha}_{2}$ plasmon-polariton polarization field in the MNP dimer.

In Fig. 2, we illustrate the avoiding effect of the coupling to a quantum-dot on the SHG process. We time evolve Eqs. (6a-6d) to obtain steady state values for the excitations.

Without the presence of a quantum object, the SHG would be maximum $\left(\tilde{\alpha}_{2}=-i \chi^{(2)} \tilde{\alpha}_{1}^{2} / \gamma_{2}\right)$ that is when the second plasmon-polariton mode is on resonance $\omega_{2}=2 \omega$ [see Eq. (9)]. In Fig. 2, we observe that even at the presence of this resonance $\left(\omega_{2}=2 \omega\right)$, EIT suppresses the SHG by 9 orders of magnitude. This effect arises simply

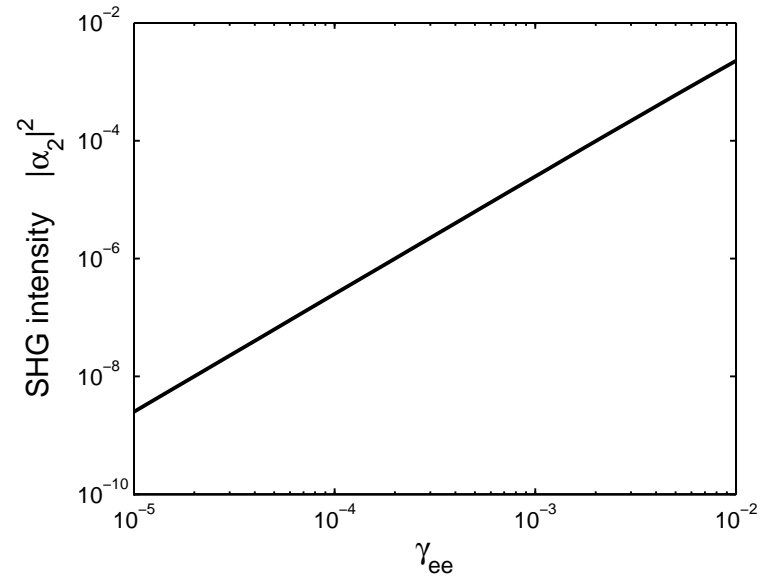

FIG. 3: The available suppression for different values of the quantum decay rate $\gamma_{e e}$. The log-log plot has a steepness of value $\simeq 2$, thus pointing out the relation $\left|\alpha_{2}\right|^{2} \sim \gamma_{e e}^{2}$. This relation can be easily inferred from Eq. (9) for small values of $\gamma_{e e}$ with $\omega_{e g}=2 \omega$. Smaller quantum decay rate results in higher quality suppression.

because EIT doesn't allow the polarization of the second plasmon-polariton mode $\hat{a}_{2}$ at $2 \omega$. The two paths -introduced in the MNP dimer due to the hybridization with the quantum dot- for polarization transfer from the $\hat{a}_{1}$ mode interfere destructively. This cancels the transfer of $\hat{a}_{1}$ polarization (oscillating at $\omega$ ) to $\hat{a}_{2}$ polarization in the dimer (oscillating at $2 \omega$ ). Fig. 3 shows the dependence of the SHG intensity $\left|\alpha_{2}\right|^{2}$ on the decay rate $\gamma_{e e}$ of the quantum emitter that is coupled to the dimer. The slope of the graph implies a $\left|\alpha_{2}\right|^{2} \sim \gamma_{e e}^{2}$ dependence. In fact, this can be easily inferred from Eq. (9) for the small values of $\gamma_{e g}$ when $\omega_{e g} \cong 2 \omega$.

\section{Enhancement of the nonlinear conversion process}

On the other hand, the interference effects can be arranged to carry the SHG process closer to the resonance. In the denominator of Eq. (9) the imaginary part of the first term $\left|f_{c}\right|^{2} y /\left[i\left(\omega_{e g}-2 \omega\right)+\gamma_{e g}\right]$ can be arranged to cancel the $i\left(\omega_{2}-2 \omega\right)$ expression in the second term of the denominator. This gives the condition

$$
\left|f_{c}\right|^{2} y\left(\omega_{e g}-2 \omega\right)+\left(\omega_{2}-2 \omega\right)\left[\left(\omega_{e g}-2 \omega\right)^{2}+\gamma_{e g}^{2}\right]=0
$$

Eq. (10) has two roots

$$
\omega_{e g}^{(1,2)}-2 \omega=\frac{\left|f_{c}\right|^{2}|y|}{\omega_{2}-2 \omega} \mp \sqrt{\frac{\left|f_{c}\right|^{4}|y|^{2}}{\left(\omega_{2}-2 \omega\right)^{2}}-4 \gamma_{e g}^{2}} .
$$

The first (smaller) root $\omega_{e g}^{(1)} \cong 2 \omega+2 \gamma_{e g}$ is not useful for SHG enhancement. Because it enlarges the real part of the $\left|f_{c}\right|^{2} y /\left[i\left(\omega_{e g}-2 \omega\right)+\gamma_{e g}\right]$ term. This is already the suppression condition for SHG. 


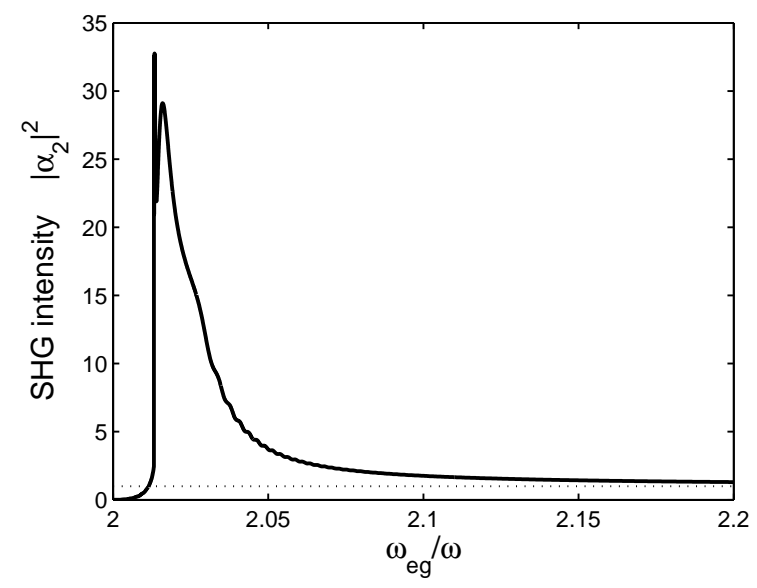

FIG. 4: The enhancement of the nonlinear process. The second PP mode $\left(\hat{a}_{2}\right)$ is far-off resonant to the SHG $\left(\omega_{2}=2.4 \omega\right)$. The nonlinear process can be carried closer to resonance by arranging the quantum level spacing [65] to $\omega_{e g} \cong 2.013 \omega$. The conversion is enhanced upto 30 times compared to the off-resonant process. The conversion for off-resonant process (with $f_{c}=0$ ) is represented by unity in the figure. For $\omega_{e g}=2 \omega$, nonlinear process is suppressed similar to Fig. 2 . Note that, the bandwidth for the enhancement of the nonlinear conversion is much wider than the suppression bandwidth. Decay rate of the nanoscale resonator $[59,60]$ is chosen as $\gamma_{2}=0.01 \omega$.

Since $\omega_{e g}$ is not very close to $2 \omega$ for the second root $\omega_{e g}^{(2)}$, it doesn't blow up the real part of the $\left|f_{c}\right|^{2} y /\left[i\left(\omega_{e g}-\right.\right.$ $\left.2 \omega)+\gamma_{e g}\right]$ term. Nevertheless, $\omega_{e g}^{(2)}$ is also the minimization condition for the absolute value of the denominator of Eq. (9), which gives rise to the maximum SHG.

For the case of the suppression of SHG, one safely use the approximation $y \cong-1$. Because, excitations are suppressed in the hybrid system, $\rho_{e e} \cong 0$, and this leads to $y=\rho_{e e}-\rho_{g g} \cong-1$. However, in the case of SHG, enhancement, one can not use the value $\cong-1$ for $y$. We observe that at resonances $y$ can attain the inversion values close to zero. However, Eq. (11) still serves at least as a guess value for the order of $\omega_{e g}^{(2)}$, where SHG enhancement arises.

In Fig 4, we depict the enhancement of the nonlinear frequency conversion for a nanoscale dimer whose plasmon-polariton excitation mode $\left(\omega_{2}=2.4 \omega\right)$ is far off-resonant to the SH frequency. Off-resonant SHG conversion $\left[f_{c}=0\right.$ in Eq. (9)] is represented by unity in Fig. 4. We observe a 30 times enhancement in the conversion intensity for the choise of $\omega_{e g}=2.013 \omega$. Parameters are given in Fig. 4. As it can be inferred from Eq. (9); enhancement efficiency can be grown much more with respect to the off-resonant value $\left(\omega_{2}=2.4 \omega\right.$ with $\left.f_{c}=0\right)$ if higher quality (small $\gamma_{2}$ ) resonators are used. Quality factors of $\sim 1300$ can be achieved for micro-cavities operating at optical wavelength $[59,60]$.

\section{Model for other nonlinearities}

If one is interested in the control of the third harmonic generation (THG) process, instead of the $\mathrm{SH}$ one, she/he would simply change Eq. (5) to

$$
\hat{H}_{t h}=\hbar \chi^{(3)}\left(\hat{a}_{2}^{\dagger} \hat{a}_{1} \hat{a}_{1} \hat{a}_{1}+\hat{a}_{1}^{\dagger} \hat{a}_{1}^{\dagger} \hat{a}_{1}^{\dagger} \hat{a}_{2}\right)
$$

In this case, Eq. (9) should simply be modified to

$$
\tilde{\alpha}_{2}=\frac{i \chi^{(3)}}{\frac{\left|f_{c}\right|^{2} y}{i\left(\omega_{e g}-3 \omega\right)+\gamma_{e g}}-\left[i\left(\omega_{2}-3 \omega\right)+\gamma_{2}\right]} \tilde{\alpha}_{1}^{3} .
$$

All of our considerations, states above, works for Eq. 13 as well. In order to be able to use the introduced model, one has to be careful if the nonlinear conversion takes place to another PP mode which is resolvable.

In some nonlinear conversion processes (e.g. enhanced Raman scattering [5]), both the drive $(\omega)$ and the generated $\left(\omega_{N L}\right)$ frequencies may excite in the same PP mode $\left(\hat{a}_{1}\right)$. This may occur because, frequency spacing $\left|\omega_{N L}=\omega\right|$ can be small compared to the decay rate $\left(\gamma_{1}\right)$ of the PP polarization mode. In this case too, suppression of the nonlinear conversion will necessarily arise for $\omega_{e g}=\omega_{N L}$. This is because; coupling to a high quality quantum oscillator prevents the polarization/absorption for frequency values around $\simeq \omega_{e g}$ independent of the details of the conversion mechanism [16, 39, 61]. The generated frequency mode will be suppressed if it coincides with $\omega_{e g}$. Fig. 5 in ref. [16] demonstrates that polarization cancellation emerges at $\omega=\omega_{e g}$ for the coupled MNP-quantum emitter system. However, enhancement of the nonlinearities depends on the physics of the conversion mechanism.

\section{DISCUSSIONS AND CONCLUSIONS}

It is well demonstrated that the presence of a quantum object, with a smaller decay rate, changes the optical response of MNPs dramatically. Due to the destructive interference of the (hybridized) absorption paths, MNP can not be polarized at the resonance frequency of the quantum emitter.

We demonstrate that a similar path interference effect can be adopted to both suppress and enhance the nonlinear conversion processes in MNP dimers: A quantum object is placed into the middle of two hybridized MNPs where hot-spot of both plasmon-polariton mode emerges. If the quantum emitter is resonant to the second or third harmonic frequency, (e.g. $\omega_{e g}=2 \omega$ ), this frequency conversion process is suppressed several orders of magnitude. Because, EIT does not allow the excitation of the $2 \omega$ oscillation in the second dimer mode $\left(\omega_{2}\right)$. On the other hand, the similar interference effects can be used also to enhance the nonlinear frequency conversion. The level spacing of the quantum emitter can be arranged [65] so that the nonresonant $\left[\right.$ e.g. $\left.\left(\omega_{2}-2 \omega\right)\right]$ terms cancel. 
We present our method for the engineering of SHG process. However, the method can be used also for other nonlinear frequency generation processes, as long as the converted frequency occurs to a plasmon-polariton mode other than the driven plasmon mode. On the other hand, suppression phenomenon is independent from the conversion mechanism. If a quantum emitter -resonant to the generated frequency- is coupled to the MNP system, the conversion process is prohibited.

\section{Acknowledgments}

D.T. and M.E.T acknowledge support from TÜBITAK-KARIYER Grant No. 112T927. We specially thank Gürsoy B. Akgüç for guiding discussions and his intensive help in the generation of Figure 1.
[1] M. I. Stockman, Opt. Express, 2011, 19, 22029-22106.

[2] X. Wu, S. K. Gray, and M. Pelton, Opt. Express, 2010, 18, 23633-23645.

[3] L. Novotny and B. Hecht, Principles of Nano-Optics, Cambridge University Press, Cambridge, 2006.

[4] M. Kauranen and A. V. Zayats, Nat. Photonics, 2012, 6, 737-748.

[5] B. Sharma, R. R. Frontiera, A. I. Henry, E. Ringe and R. P. Van Duyne, Mater. Today, 2012, 15, 16-25.

[6] P. Genevet, J.-P. Tetienne, E. Gatzogiannis, R. Blanchard, M. A. Kats, M. O. Scully, and F. Capasso, Nano Lett., 2010, 10, 4880-4883.

[7] S. Wunderlich and U. Peschel, Opt. Express, 2013, 21, 18611-18623.

[8] S. Gao, K. Uneo and H. Misawa, Accounts Chem. Res., 2011, 44, 251-260.

[9] M. S. Singh, Nanotechnology, 2013, 24, 125701.

[10] G. F. Walsh and L. D. Negro, Nano Lett., 2013, 13, 31113117.

[11] K. Kneipp, Y. Wang, H. Kneipp, L. T. Perelman, I. Itzkan, R. R. Dasari, and M. S. Feld, Phys. Rev. Lett., 1997, 78, 1667-1670.

[12] C.-H. Hsieh, L.-J. Chou, G.-R. Lin, Y. Bando, and D. Golberg, Nano Lett., 2008, 8, 3081-3085.

[13] H. Atwater and A. Polman, Nature Mat., 2010, 9, 205213

[14] W. Li, A. K. Tuchman, H.-C. Chien and M. A. Kasevich, Phys. Rev. Lett., 2007, 98, 040402.

[15] G.-B. Jo, Y. Shin, S. Will, T. A. Pasquini, M. Saba, W. Ketterle, D. E. Pritchard, M. Vengalattore and M. Prentiss, Phys. Rev. Lett., 2007, 98, 030407.

[16] M. E. Taşgın, Nanoscale, 2013, 5, 8616-8624.

[17] E. Altewischer, M. P. van Exter, and J. P. Woerdman, Nature, 2012 , 418, 304-306.

[18] A. R. Chraplyvy, J. Lightwave Technol., 1990, 8, 15481557.

[19] A. M. Glass, D. J. DiGiovanni, T. A. Strasser, A. J. Stentz, R. E. Slusher, A. E. White, A. R. Kortan and B. J. Eggleton, Bell Labs Tech., 2000, January - March, 168-187.

[20] J. Toulouse, Lightwave Technol., 2005, 23, 3625-2641.

[21] L. G. L. Wegener, M. L. Povinelli, A. G. Green, P. P. Mitra, J. B. Stark and P. B. Littlewood, Physica D, 2005, 189, 81-99.

[22] J. Wiersiq, Phys. Rev. A, 2006, 73, 031802.

[23] S. B. Lee, J. Yang, S. Moon, S. Y. Lee, J. B. Shim, S. W. Kim, J. H. Lee, K. An, arXiv:0905.4478v2, 2009.

[24] S. H. Strogatz, Nonlinear Dynamics and Chaos, Perseus Books Publishing L. L. C., New York, 2003.

[25] M. O. Scully and M. S. Zubairy, Quantum Optics, Cam- bridge University Press, Cambridge, 1997.

[26] A. Manjavacas, J. GarcÃa de Abajo and P. Nordlander, Nano Lett., 2011, 11, 2318-2323.

[27] P. Weis, J. L. Garcia-Pomar, R. Beigang and M. Rahm, Opt. Express, 2011, 19, 23573-23580.

[28] S. G. Kosionis, A. F. Terzis, S. M. Sadeghi and E. Paspalakis, J. Phys. Condens. Matter, 2013, 25, 045304.

[29] R. D. Artuso and G. W. Bryant, Phys. Rev. B, 2010, 82, 195419.

[30] R. D. Artuso and G. W. Bryant, Nano Lett., 2008, 8, 2106-2111

[31] E. Waks and D. Sridharan, Phys. Rev. A, 2010, 82, 043845.

[32] A. Ridolfo, O. D. Stefano, N. Fina, R. Saija, and S. Savasta, Phys. Rev. Lett., 2010, 105, 263601.

[33] W. Zhang, A. O. Govorov and G. W. Bryant, Phys. Rev. Lett., 2006, 97, 146804

[34] S. G. Kosionis, A. F. Terzis, V. Yannopapas and E. Paspalakis, J. Phys. Chem. C, 2012, 116, 23663-23670.

[35] M. A. Noginov, G. Zhu, A. M. Belgrave, R. Bakker, V. M. Shalaev, E. E. Narimanov, S. Stout, E. Herz, T. Suteewong and U. Wiesner, Nature, 2009, 490, 1110-1112.

[36] M. Pfeiffer, K. Lindfors, C. Wolpert, P. Atkinson, M. Benyoucef, A. Rastelli, O. G. Schmidt, H. Giessen and M. Lippitz, Nano Lett., 2010, 10, 4555-4558.

[37] L. Zhao, T. Ming, H. Chen, Y. Liang and J. Wang, Nanoscale, 2011, 3, 3849-3859.

[38] P. Anger, P. Bharadwaj and L. Novotny, Phys. Rev. Lett., 2006, 96, 113002.

[39] C. L. G. Alzar, M. A. G. Martinez and P. Nussenzveig, Am. J. Phys., 2002, 70, 37-41.

[40] P. Nordlander, C. Oubre, E. Prodan, K. Li and M. I. Stockman, Nano Lett., 2004, 4, 899-903.

[41] A. G. Curto, G. Volpe, T.H. Taminiau, M. P. Kreuzer, R. Quidant and N. F. van Hulst, Science, 2010, 329, 930-933

[42] S. J. Barrow, X. Wei, J. S. Baldauf, A. M. Funston and P. Mulvaney, Nature Comm., 2012, 30, 1275.

[43] N. B. Grosse, J. Heckmann, and U. Woggon, Phys. Rev. Lett., 2012, 108, 136802.

[44] C. Ciraci, E. Poutrina, M. Scalora and D. R. Smith, Phys. Rev. B, 2012, 85, 201403.

[45] M. Finazzi and F. Ciccacci, Phys. Rev. B, 2012, 86, 035428.

[46] M. I. Dykman, D. G. Luchinsky, R. Mannella, E. McClintock, M. Soskin and N. D. Stein, Phys. Rev. E, 1996, 54, 2366-2377.

[47] G. Y. Panasyuk, J. C. Schotland and V. A. Markel, Phys. Rev. Lett., 2008, 100, 047402.

[48] W. Hübner, K. H. Bennemann and K. Böhmer, Phys. 
Rev. B, 1994, 50, 17597-17605.

[49] S. Kielich, R. Tanas and R. Zawodny, J. Mod. Optic., 1987, 34, 979-996.

[50] G. Bachelier, J. Butet, I. Russier-Antoine, C. Jonin, E. Benichou and P. F. Brevet, Phys. Rev. B, 2010, 82, 235403.

[51] A. T. Georges, J. Opt. Soc. Am. B, 2011, 28, 1603-1606.

[52] G. Y. Panasyuk, Phys. Rev. B, 2011, 84, 155460.

[53] A. Trügler, Dissertation 2011.

[54] A. Bouhelier, R. Bachelot, G. Lerondel, S. Kostcheev, P. Royar, P. Wiederrecht, Phys. Rev. Lett., 2005, 95, 267405.

[55] M. R. Beversluis, A. Bouhelier and L. Novotny, Phys. Rev. B, 2003, 68, 115433.

[56] P. Mühlschlegel, H.-J. Eisler, O. J. F. Martin, B. Hecht and D. W. Pohl, Science, 2005, 308, 1607-1609.

[57] In example, Enhanced Yellow Fluorescent Protein (EYFP) does not have a SHG $(532 \mathrm{~nm})$ response for illumination at $1064 \mathrm{~nm}$ wavelength.

[58] L. Mandel and E. Wolf, Optical Coherence and Quantum Optics, Cambridge University Press, Cambridge, 1995.

[59] B. Min, E. Ostby, V. Sorger, E. Ulin-Avila, L. Yang, X.
Zhang and K. Vahala, Nature Lett., 2009, 457, 455-459.

[60] P. R. West, S. Ishii, G. V. Naik, N. K. Emani, V. M. Shalaev and A. Boltasseva, Laser Photonics Rev., 2010, 4, 795-808.

[61] P. Tassin, L. Zhang, R. Zhao, A. Jain, T. Koschny and C. M. Soukoulis, Phys. Rev. Lett., 2012, 109, 187401.

[62] We note that hot-spots of both modes occurs in the middle of the two MNPs [63].

[63] N. J. Halas, S. Lal, W.-S. Chang, S. Link, and P. Nordlander, Chem. Rev., 2011, 111, 3913.

[64] Use of resonators with high quality behavior ( $Q=1300$ in ref $[59,60])$ can provide narrower bands for suppression/enhancement of frequency conversion.

[65] In practice, arrangement of the level spacing of a quantum emitter $\left(\omega_{e g}\right)$ to high accuracy may not be possible for experimental demonstrations. However one may arrange the incident frequency $\omega$ such that $2 \omega$ coincides with the desired level spacing.

[66] J. Butet, J. Duboisset, G. Bachelier, I. Russier-Antoine, E. Benichou, C. Jonin and P. F. Brevet, Nano Lett., 2010, 10, 1717-1721. 